\title{
The performance of armour steels with pre-layers against fragment simulating projectiles
}

\author{
Rogier van der Wal $^{1, *}$, Erik Carton $^{1}$, Frits Hilvers ${ }^{1}$ \\ ${ }^{1}$ TNO Defence, Safety and Security, Laboratory for Ballistics Research, Ypenburgse Boslaan 2, 2496 ZA Den Haag, The \\ Netherlands
}

\begin{abstract}
Armour steels have proven to be promising solutions for protection against fragments from threat weapons. The area to cover for ship bulkheads is usually large, so cost of the raw material is an important driver. For new ships, additional mass can be compensated for in the design. Past research by $\mathrm{TNO}$ and other workers has shown that the ballistic limit $\mathrm{V}_{50}$ of armour steels against fragments is increased significantly by adding a front or pre-layer. This layer can be a variety of materials ranging from cardboard to glass. In TNO's Laboratory for Ballistics Research a test program was conducted to study the effect of high pressure laminate, polymer and fire insulation pre-layers at the $\mathrm{V}_{50}$ and well above the ballistic limit. The high velocities are typically associated with fragments from relevant threats for warships. Fragment simulating projectiles were fired on armour steel plates of varying type and thickness with these pre-layers and measured the residual velocity and resulting hole sizes. The tests resulted in clear dependencies of the residual velocity as a function of impact velocity and pre-layer (type and thickness). Analysis of the data showed that there are several counteracting effects interacting when a pre-layer is applied to armour steel. The failure mechanism of the steel as well as the hardness and thickness of the pre-layer seem to influence the outcome of this interaction and hence the response of the steel to various pre-layers. The results of this research will be used in the design of fragment and blast resistant bulkheads for future naval ships.
\end{abstract}

\section{Introduction}

One of the most important considerations in increasing naval ship resilience against threats is the confinement of damage, particularly in longitudinal direction of the ship. Researchers are exploring new technologies to protect against fragments of internally exploding shells or missiles [1].

Within the HARDCORE project a consortium of industry partners, Netherlands' Ministry of Defence and TNO have assessed the feasibility of pre-layers on armour steel for the application of fragment protection in naval ships [2].

The research showed that this technology is promising for the development of medium weight, affordable fragment protection. The successor project HARNESS aims to widen the scope to confine additional damage effects, particularly retaining fire resistance after an internal explosion and increasing the technology readiness level. HARNESS is short for Hybrid ARmour for iNternal Explosions on Surface Ships. Partners in HARNESS are:

- Netherlands' Ministry of Defense - Defense Materiel Organisation,

- Federal Office of Bundeswehr Equipment, Information Technology and In-Service Support (BAAINBw),

- Damen Schelde Naval Shipbuilding,
- ThyssenKrupp Marine Systems,

- Swedish Steel AB,

- TNO Defence Research.

\section{Background}

In $[3,4,5]$ co-workers observed a significant increase of the ballistic limit $\mathrm{V}_{50}$ when putting an additional elastomeric layer on the impact side of high hardness steels. This will be referred to as a pre-layer. The layer must be touching the steel target. $\mathrm{V}_{50}$ is defined as the average of an equal number of highest partial penetration velocities $^{\mathrm{a}}$ and the lowest complete penetration velocities which occur within a specified velocity spread [6]. In [2] the physics behind this phenomenon were further investigated by varying the type of pre-layer. The research was however focussed on the effect near the $\mathrm{V}_{50}$, at velocities much lower than fragments from actual threats. For the design of fragment resistant ship

\footnotetext{
${ }^{a}$ A complete penetration occurs when the impacting projectile, or any fragment thereof, or any fragment of the test specimen perforates the witness plate, resulting in a crack or hole which permits light passage when a 60 -watt, 110 -volt bulb is placed proximate to the witness plate. Any impact which is not a complete penetration shall be considered a partial penetration. $[6]$.
}

Corresponding author: rogier.vanderwal@tno.nl 


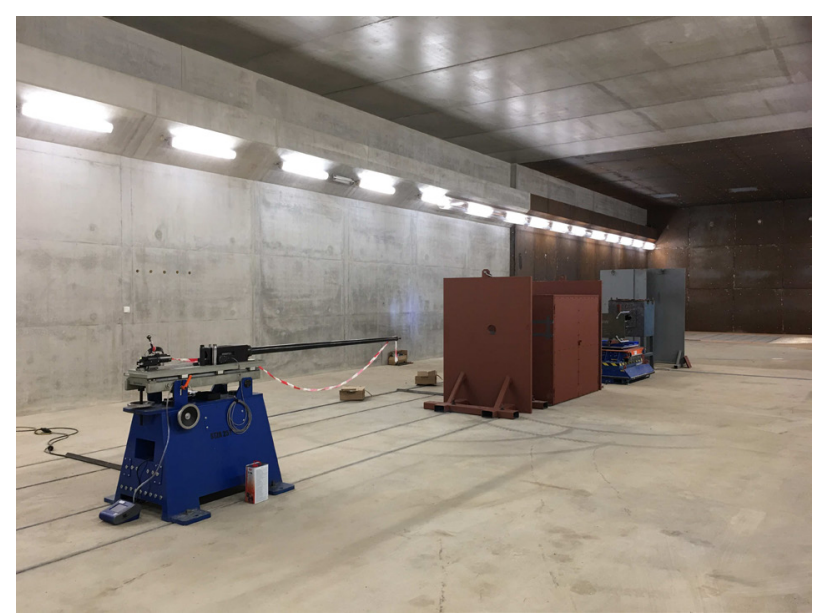

Fig. 1. Multipurpose test hall with setup for ballistic trials.

bulkheads it is required to investigate the effect of the pre-layer at higher impact velocities.

\section{Objective}

The objective of this research is to assess and understand the effect of a pre-layer on armour steels at velocities near the ballistic limit $V_{50}$ of the pre-layer/armour combination and well above the $\mathrm{V}_{50}$.

\section{Test setup}

\subsection{Ballistic range}

Ballistic trials where done in the brand new multipurpose facility of TNO's Laboratory for Ballistics Research. Fig. 1 shows an overview of the test hall with:

- Gun,

- Velocity and orientation measurement equipment (inside the brown enclosure),

- Target on a remote controlled manipulator.

Visible on the photograph in Fig. 2 is the high speed camera filming perpendicular to the shooting direction behind the target. This is used for determining the residual velocity of the threat. The threat is a fragment simulating projectile (FSP) as defined in [7].

\subsection{Target configurations}

For the target armour plates two types of armour steel with varying hardness were selected. The pre-layers where chosen on the basis of hardness, thickness and applicability in ships. Table 1 shows the tested configurations. ARMOX and RAMOR are SSAB branded armour steel types. The type number refers to the Brinell hardness in HBW. Most important mechanical properties for this study are listed in Table 2.
Table 1. Armour steels and pre-layers.

\begin{tabular}{|c|l|l|}
\hline Concept & Pre-layer & Armour steel \\
\hline 1 & none & $10.2 \mathrm{~mm}$ ARMOX 600T \\
\hline 2 & $3 \mathrm{~mm}$ HPL & $10.2 \mathrm{~mm}$ ARMOX 600T \\
\hline 3 & $6 \mathrm{~mm}$ HPL & $10.2 \mathrm{~mm}$ ARMOX 600T \\
\hline 4 & $10 \mathrm{~mm}$ PRMGRD & $10.2 \mathrm{~mm}$ ARMOX 600T \\
\hline 5 & none & $10.2 \mathrm{~mm}$ RAMOR 450 \\
\hline 6 & $6 \mathrm{~mm}$ HPL & $10.2 \mathrm{~mm}$ RAMOR 450 \\
\hline 7 & $4 \mathrm{~mm}$ PU D60 & $10 \mathrm{~mm}$ RAMOR 450 \\
\hline 8 & $6 \mathrm{~mm}$ PU A80 & $10.2 \mathrm{~mm}$ RAMOR 450 \\
\hline 9 & none & $5.5 \mathrm{~mm}$ ARMOX 600T \\
\hline 10 & $6 \mathrm{~mm}$ HPL & $5.5 \mathrm{~mm}$ ARMOX 600T \\
\hline
\end{tabular}

PRMGRD is short for Promat PROMAGUARD ${ }^{\circledR}$ passive fire protection. HPL stands for high pressure laminate, a material used for decorative finishing of compartment dividers. The density and hardness of our HPL test samples was $1488 \mathrm{~kg} / \mathrm{m}^{3}$ and D93, respectively. PU denotes polyurea with the type number referring to the Shore hardness. Density of the A80 type is 936 $\mathrm{kg} / \mathrm{m}^{3}$, of the D60 type $1080 \mathrm{~kg} / \mathrm{m}^{3}$. The polyurea was manufactured by The Polyurea Factory in Zeeland, The Netherlands. Setup of the test sample on the target manipulator is shown in Fig. 2.

Table 2. Mechanical properties for ARMOX 600T and RAMOR 450.

\begin{tabular}{|l|c|c|c|c|}
\hline Brand & Type & HBW & $\begin{array}{c}\text { Yield } \\
\text { strength } \\
\text { min (MPa) }\end{array}$ & $\begin{array}{c}\text { Tensile } \\
\text { strength } \\
\text { (MPa) }\end{array}$ \\
\hline ARMOX & $600 \mathrm{~T}$ & $570-640$ & $1500^{*}$ & $2000^{*}$ \\
\hline RAMOR & 450 & $400-480$ & 1000 & 1150 \\
\hline
\end{tabular}

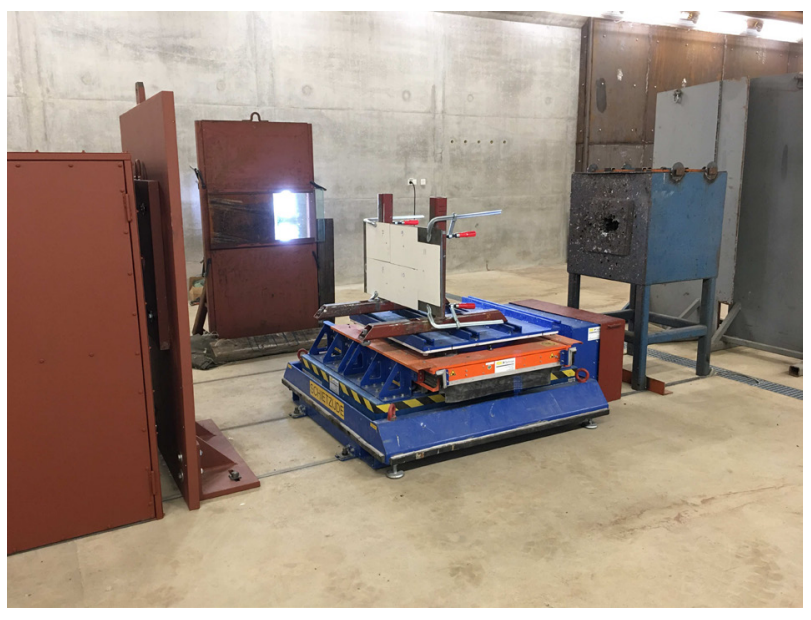

Fig. 2. $10.2 \mathrm{~mm}$ ARMOX 600T with $6 \mathrm{~mm}$ HPL pre-layer on the target manipulator. Behind the lighted glass panel is the high speed camera. 


\subsection{Measurements}

The following parameters were measured for each shot:

- Impact velocity,

- Yaw angle of the impacting FSP,

- Residual velocity using the high speed camera and Tracker software,

- Resulting hole sizes in the armour plates for complete penetrations ,

- For some FSP's the residual mass was determined. As FSPs were not collected for each shot, they could not be linked to individual shots.

\section{Results}

On concept 1-8 a total of 41 shots were fired under 0 NATO. The residual velocities (also obtained from the HS video images) can be seen in the graph of

Fig. 3. This is a representation commonly used in ballistics and shows the residual velocity of the threat as a function of the impact velocity. The (blue) solid lines connect the markers of the ARMOX 600T armour configurations. The marker is an indicator for the type of pre-layer as given in the legend. The dashed (orange) lines show the results for the tests on RAMOR 450 with varying pre-layers. The error in measuring the impact and residual velocity is about $\pm 10 \mathrm{~m} / \mathrm{s}$, slightly smaller than the size of the marker.

From

Fig. 3 it can be observed that in this impact velocity range bare ARMOX 600T and RAMOR 450 perform comparably. When adding the $6 \mathrm{~mm}$ HPL pre-layer, the drop in residual velocity is about $100 \mathrm{~m} / \mathrm{s}$ in the high velocity regime. This is comparable for both ARMOX 600T and RAMOR 450. One partial penetration at 954 $\mathrm{m} / \mathrm{s}$ impact velocity for ARMOX 600T with the $6 \mathrm{~mm}$ HPL pre-layer can be observed. Here the delta with the bare plate is nearly $500 \mathrm{~m} / \mathrm{s}$. A similar effect is visible with the thinner $3 \mathrm{~mm}$ HPL pre-layer. There is a delta of about $40 \mathrm{~m} / \mathrm{s}$ in residual velocity when comparing the bare ARMOX 600T plate with the $3 \mathrm{~mm}$ HPL prelayered plate. A partial penetration at $777 \mathrm{~m} / \mathrm{s}$ of the prelayered plate occurred and a $334 \mathrm{~m} / \mathrm{s}$ perforation of the bare plate at $765 \mathrm{~m} / \mathrm{s}$ impact velocity.

The RAMOR 450 plate with the $6 \mathrm{~mm}$ pre-layer shows one partial penetration at $776 \mathrm{~m} / \mathrm{s}$, well below the $954 \mathrm{~m} / \mathrm{s}$ of the ARMOX 600T plate with the same prelayer. From this it may be concluded that the harder ARMOX 600T with a high yield strength benefits more from a pre-layer than the softer, more ductile RAMOR 450 at impact velocities near its $V_{50}$. At high velocities the difference is small.

Fig. 4 gives the hole sizes in the armour plates as a function of impact velocity. The hole size was determined by measuring the smallest and largest diameter and assuming an elliptical shape. In many cases the diameters were comparable resulting in round holes. On the vertical axis of Fig. 4 is the area of the hole, given as a fraction of the FSP impact area. The hole size (area) increased almost linearly with the impact velocity. The bare RAMOR 450 plate gives on average about $10 \%$ larger holes compared to the ARMOX 600T.

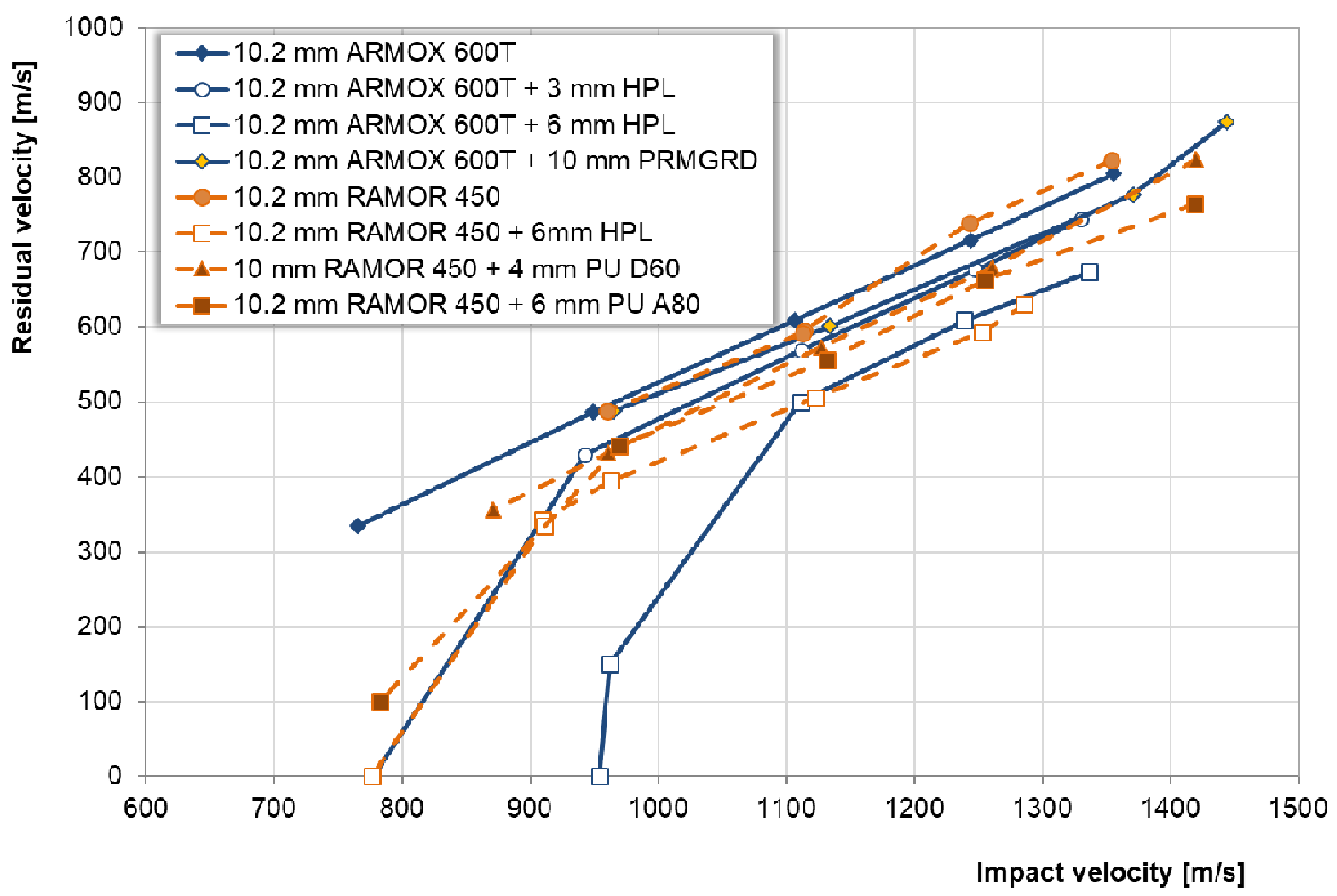

Fig. 3. Results of 41 shots on armour steels with varying types of pre-layer and bare armour for reference. The graph gives the residual velocity of the threat as a function of impact velocity. Lines connect the dots. The residual value of $150 \mathrm{~m} / \mathrm{s}$ for the shot with impact velocity of $962 \mathrm{~m} / \mathrm{s}$ is an estimate of the plug velocity. The FSP did not penetrate completely. 


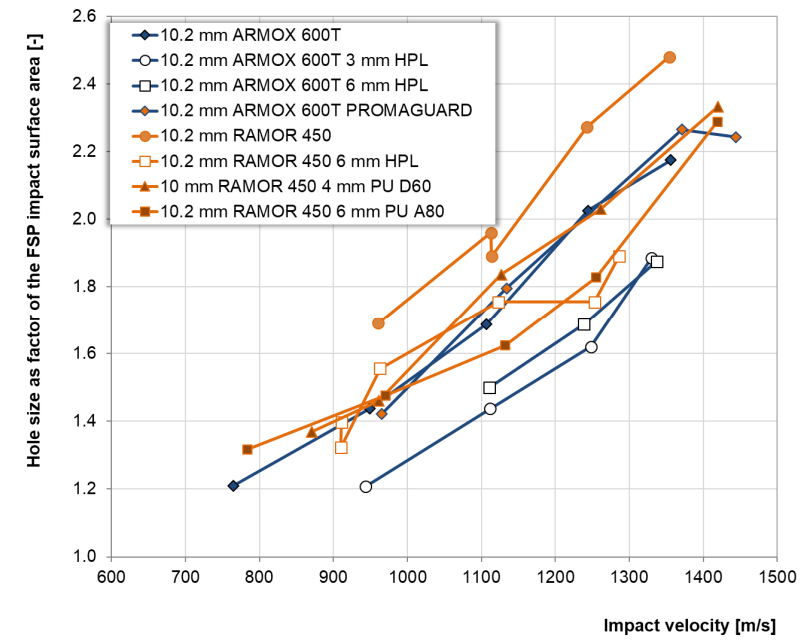

Fig. 4. Hole sizes resulting from complete penetrations of an FSP for bare armour plates and with a pre-layer.

The application of a pre-layer resulted in a decrease in hole size (area) of about $20 \%$ for both steel types.

In order to address the influence of the thickness of the armour plate on the efficiency of the pre-layer, Concepts 9 and 10 were tested. Thickness of the ARMOX 600T plate is about half that of the plates in Concept 1-4.

Two shots were done on the bare plate and two shots on the plate with a $6 \mathrm{~mm}$ HPL pre-layer at around 1600 $\mathrm{m} / \mathrm{s}$. The results are shown in Fig. 5. The delta between the shots with a pre-layer compared with that without a pre-layer is about $95 \mathrm{~m} / \mathrm{s}$. The effect on the $10.2 \mathrm{~mm}$ plate was just over $100 \mathrm{~m} / \mathrm{s}$ in the range $1100-1350 \mathrm{~m} / \mathrm{s}$, as discussed previously.

Fig. 6 shows two typical high speed video stills showing the expanding fragment cloud behind a $5.5 \mathrm{~mm}$ bare ARMOX 600T target. The top image shows shattering of the FSP resulting in a cloud of particles behind the bare plate. The bottom image is the result of a comparable shot on a $5.5 \mathrm{~mm}$ ARMOX 600T plate with a $6 \mathrm{~mm}$ HPL pre-layer. On the center line and to the left of the image the plug and deformed FSP are visible.

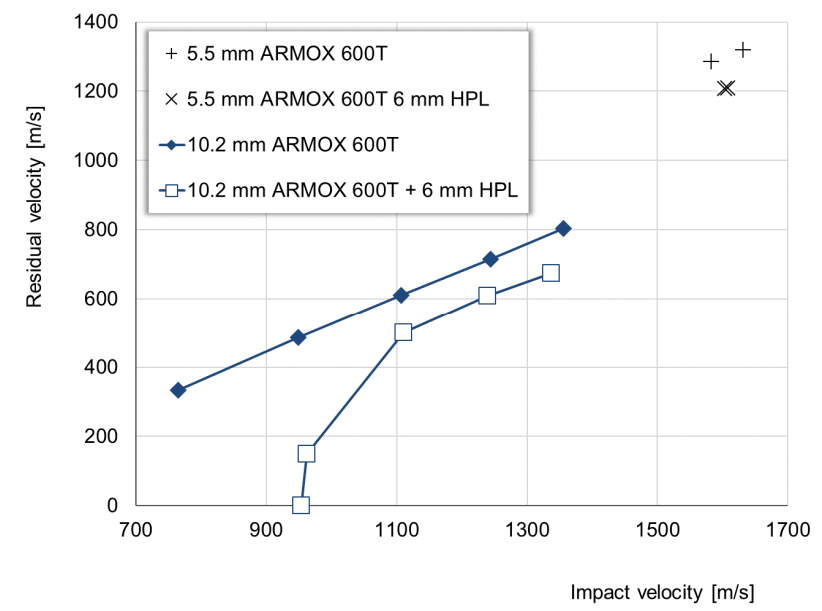

Fig. 5. Shots on $5.5 \mathrm{~mm}$ ARMOX $600 \mathrm{~T}$ with and without a 6 mm HPL pre-layer. Lines connect the markers.
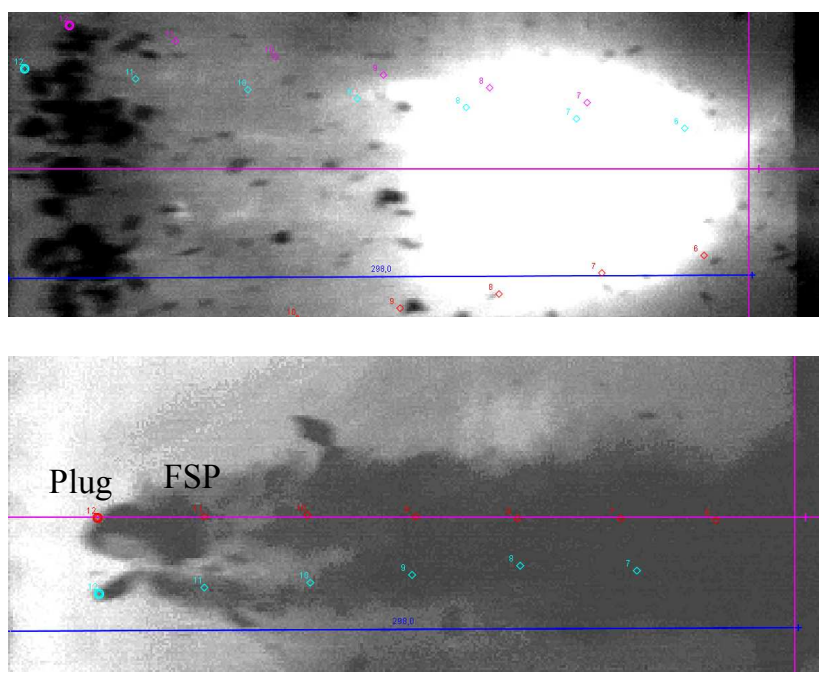

Fig. 6. Video still of expanding fragment clouds behind a bare steel plate (top) and a pre-layered plate (bottom).

\section{Analysis}

Using the residual velocity and the mass of the fragments (from the FSP and target plate), the kinetic energy behind the target plate can be calculated. The kinetic energy density directly behind the plate can then be obtained by dividing by the hole area (using the size of the hole in the target plate). This can be done for all impact velocities that lead to penetrations (hence residual velocities) both for bare steel target and plates with pre-layers. Fig. 7 shows the energy density as function of the impact velocity of the FSP for both steel types with and without pre-layers.

Both for the bare plates and plates with pre-layer the energy density increases linearly with the impact velocity. However, the energy density is significantly reduced by the pre-layers. This reduction is largest for the $6 \mathrm{~mm}$ HPL pre-layer which can be both due to its higher density and hardness compared with polyurea. The reduction in energy density is mainly caused by the reduction in residual velocity of the fragments (the pre-

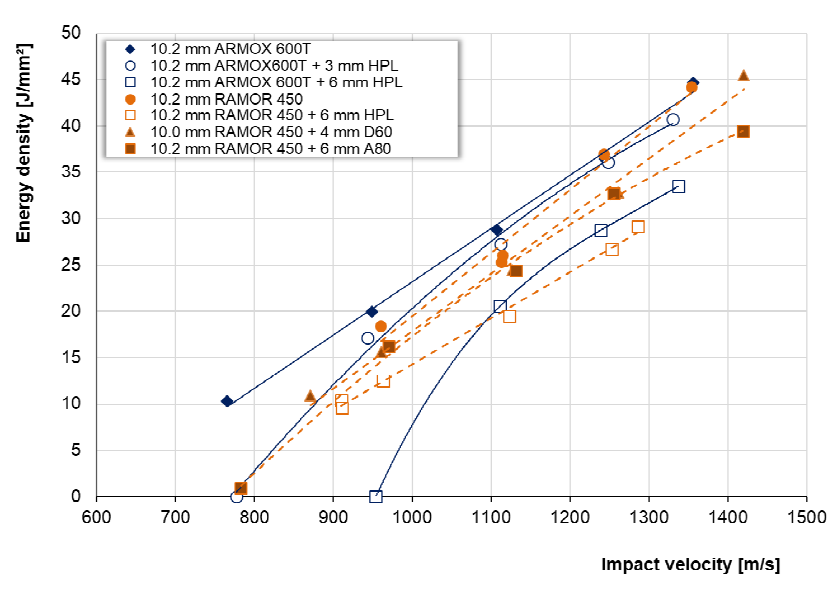

Fig. 7. Initial energy density of fragment clouds behind RAMOR 450 plates and ARMOX 600T with and without prelayers. Lines are trendlines from MS Excel. 
layers resulted in somewhat smaller hole sizes, see Fig. 4).

From the HS images (see Fig. 6) not only the residual velocity but also the radial expansion of the fragment cloud can be obtained. When the fragment cloud expands radially the kinetic energy density is reduced, as the cloud area has a squared relation with its radius. Fig. 8 shows the reduction in energy density of the fragment cloud versus distance behind the target (a $5.5 \mathrm{~mm}$ ARMOX 600T plate). The solid line shows the result for the bare steel target which initially has the largest energy density. However, due to the expansion of the cloud the ED reduces quite fast. The target plate with a $6 \mathrm{~mm}$ HPL pre-layer initially has a lower ED. However, due to the reduced spreading of the fragments, its reduction in ED with distance behind the plate is much smaller. This means that in a multi-layer armour concept the second plate may experience a higher ED due to a pre-layer on the first plate compared to a bare steel first plate, when the gap between the plates is too large. In this case the cross-over distance is about 40 $\mathrm{mm}$. Therefore, for spaced arrays a pre-layer only increases the efficiency when the plate distance is smaller than the cross-over distance of $40 \mathrm{~mm}$. In case more room is available, the use of a pre-layer on the impact side of the spaced array is not recommended. The last plate of the spaced array may benefit from a prelayer on the inside as it can increase the $\mathrm{V}_{50}$ of that plate.

\section{Discussion}

The largest effect of the pre-layer is a significant increase in the $V_{50}$ of certain steels. The impact velocities used in this work are mainly above the $V_{50}$ of the targets and the pre-layers resulted in a reduction of the residual velocity, see

Fig. 3 and Fig. 5. Although a drop in residual velocity of $100 \mathrm{~m} / \mathrm{s}$ may seem only a small effect, the kinetic energy has a squared relation with velocity. This means that the pre-layers have increased the energy dissipation of the projectile-target interaction. A reduction in residual velocity from $600 \mathrm{~m} / \mathrm{s}$ to $500 \mathrm{~m} / \mathrm{s}$ means a decrease in residual kinetic energy (of the fragments) with $44 \%$.

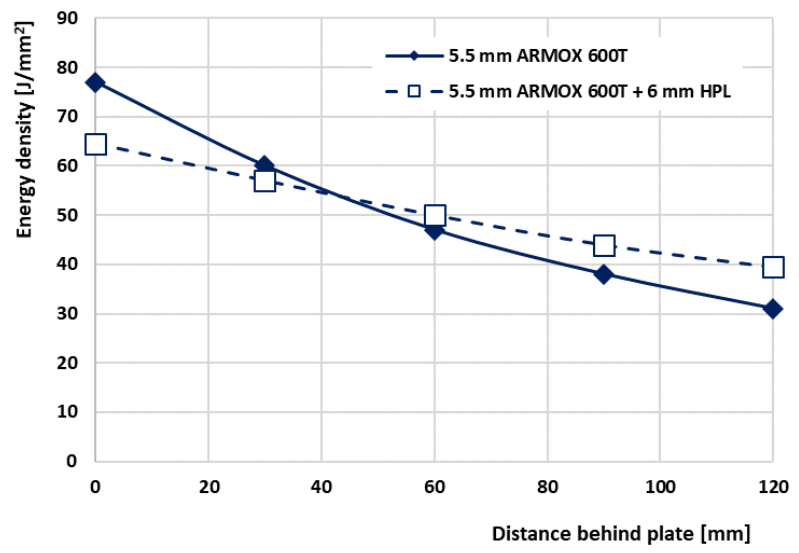

Fig. 8. Energy density of fragment clouds behind a bare 5.5 $\mathrm{mm}$ ARMOR 600T plate and with a $6 \mathrm{~mm}$ HPL pre-layer.
Adding a pre-layer on steel has several counteracting consequences:

- Failure mechanism of steel may change from early plugging to denting. This is a positive effect for the ballistic performance of steel plate,

- The effective diameter of FSP increases. This is a negative effect when regarding plug formation,

- Areal density of the target increases, which reduces the residual velocity. This is a positive effect,

- Level of fragment shatter can reduce. This is a negative effect.

Net result of these effects is not always positive and may result in a decrease in $\mathrm{V}_{50}$ for thin pre-layers on ductile steels and spaced arrays (if not designed carefully).

\section{Conclusions}

In this research a thickness effect of the pre-layer was found. Increasing the thickness of the HPL from 3 to 6 $\mathrm{mm}$ reduces the residual velocity with about $40 \mathrm{~m} / \mathrm{s}$ respectively $100 \mathrm{~m} / \mathrm{s}$. Furthermore an effect was observed of the hardness or strength of the armour steel when comparing the partial penetrations of $6 \mathrm{~mm} \mathrm{HPL}$ on either ARMOX 600T and RAMOR 450. A significant effect of ARMOX 600T plate thickness on the efficiency of the pre-layer was not observed.

Adding a pre-layer on steel has several counteracting effects. Largest effect on high hard steel types is a significant increase in $\mathrm{V}_{50}$ (for FSP threats), which is a result of a change in failure mechanism of the steel plate from plugging to denting. However, if the change in failure mechanism does not occur, the $\mathrm{V}_{50}$ may reduce as the FSP will get an increased effective diameter with adding a pre-layer. Another effect of adding a pre-layer is a reduction in energy density (ED) of the fragment cloud. However, the pre-layer also reduces the expansion of this cloud and thereby hinders the reduction of the ED with distance behind the plate. At a certain distance the ED of a bare steel plate will be lower than that of a plate with pre-layer.

Therefore, the net result of these effects is not always positive and may result in a decrease in $\mathrm{V}_{50}$ for thin prelayers on ductile steels and spaced arrays (if not designed carefully).

\section{References}

1. D. Li, H. Hou, C. Chen, X. Zhu, M. Li, Q. Yi, Experimental study on the combined damage of multi-layered composite structures subjected to close-range explosion of simulated warheads, International Journal of Impact Engineering 114, 133-146 (2018)

2. R. van der Wal, E.P. Carton, J.M. Parent, Performance enhancement of armour steel against blunt projectiles using pre-layers, Light Weight Armour Group Conference (2013) 
3. C.M. Roland, D. Fragiadakis, R.M. Gamache. Elastomer-steel laminate armor, Composite Structures 92 1059-1064 (2010)

4. B. Dyatkina, R.M. Gamacheb, Ballistic performance of front-facing fluoropolymer-coated armor composites, International Journal of Impact Engineering 114 63-68 (2018)

5. P.H. Mott, C. Giller, D. Fragiadakis, D. Rosenberg, C. Roland, Deformation of polyurea: Where does the energy go?, Polymer Physics, 105, 227-233 (1016)
6. Department Of Defense Test Method Standard, V50 Ballistic Test For Armor, MIL-STD-662F (18 December 1997)

7. AEP-2920, Procedures For The Evaluation And Classification Of Personal Armour, Bullet And Fragmentation Threats, Nato Standard, Edition A Version 1 (June 2015) 